\title{
Noncommutative-Geometry Wormholes with Isotropic Pressure
}

\author{
Peter K. F. Kuhfittig \\ Department of Mathematics, Milwaukee School of Engineering, Milwaukee, WI 53202-3109, USA
}

\begin{abstract}
The strategy adopted in the original Morris-Thorne wormhole was to retain complete control over the geometry at the expense of certain engineering considerations. The purpose of this paper is to obtain several complete wormhole solutions by assuming a noncommutative-geometry background with a concomitant isotropic pressure condition. This condition allows us to consider a cosmological setting with a perfect-fluid equation of state. An extended form of the equation generalizes the first solution and subsequently leads to the generalized Chaplygin gas model and hence to a third solution. The solutions obtained extend several previous results. This paper also reiterates the need for a noncommutative-geometry background to account for the enormous radial tension that is
\end{abstract} a characteristic of Morris-Thorne wormholes.

Keywords: traversable wormholes, noncommutative geometry, isotropic pressure

DOI: 10.31526/LHEP.2021.190

\section{INTRODUCTION}

Wormholes are tunnel-like structures in spacetime that connect widely separated regions of our Universe or different universes altogether. Although not entirely new, a detailed analysis of traversable wormholes was first performed by Morris and Thorne [1] in 1988. They had proposed the following static and spherically symmetric line element for a wormhole spacetime:

$$
d s^{2}=-e^{2 \Phi(r)} d t^{2}+\frac{d r^{2}}{1-b(r) / r}+r^{2}\left(d \theta^{2}+\sin ^{2} \theta d \phi^{2}\right)
$$

using units in which $c=G=1$. Here, $b=b(r)$ is called the shape function and $\Phi=\Phi(r)$ is called the redshift function; $\Phi(r)$ must be everywhere finite to avoid an event horizon. The shape function must also have certain properties, including the fixed-point property $b\left(r_{0}\right)=r_{0}$, where $r=r_{0}$ is the radius of the throat of the wormhole. An important requirement is the flareout condition at the throat: $b^{\prime}\left(r_{0}\right)<1$, while $b(r)<$ $r$ near the throat. For the wormhole spacetime as a whole, the most important physical property is asymptotic flatness, which demands that $\lim _{r \rightarrow \infty} \Phi(r)=0$ and $\lim _{r \rightarrow \infty} b(r) / r=0$.

The flare-out condition refers to the tunnel-like shape of $b(r)$ when viewed, for example, in an embedding diagram [1]. This condition can only be met by violating the null energy condition (NEC)

$$
T_{\alpha \beta} k^{\alpha} k^{\beta} \geq 0
$$

for all null vectors $k^{\alpha}$, where $T_{\alpha \beta}$ is the energymomentum tensor. The matter that violates the NEC is called "exotic" in [1]. In particular, for the outgoing null vector $(1,1,0,0)$, the violation has the form

$$
T_{\alpha \beta} k^{\alpha} k^{\beta}=\rho+p_{r}<0 .
$$

Here, $T_{t}^{t}=-\rho$ is the energy density, $T_{r}^{r}=p_{r}$ is the radial pressure, and $T_{\theta}^{\theta}=T_{\phi}^{\phi}=p_{t}$ is the lateral pressure.

Regarding the theoretical construction of a wormhole, Morris and Thorne adopted the following strategy: specify the functions $b=b(r)$ and $\Phi=\Phi(r)$ to produce the desired geometric properties. This strategy retains complete control over the geometry but leads to enormous practical problems: the members of the engineering team must manufacture or search the Universe for matter or fields that yield the required energymomentum tensor. There are theoretical problems as well, as can be seen from the Einstein field equations, listed next.

$$
\begin{aligned}
\rho(r)= & \frac{b^{\prime}}{8 \pi r^{2}} \\
p_{r}(r)= & \frac{1}{8 \pi}\left[-\frac{b}{r^{3}}+2\left(1-\frac{b}{r}\right) \frac{\Phi^{\prime}}{r}\right], \\
p_{t}(r)= & \frac{1}{8 \pi}\left(1-\frac{b}{r}\right)\left[\Phi^{\prime \prime}-\frac{b^{\prime} r-b}{2 r(r-b)} \Phi^{\prime}+\left(\Phi^{\prime}\right)^{2}\right. \\
& \left.+\frac{\Phi^{\prime}}{r}-\frac{b^{\prime} r-b}{2 r^{2}(r-b)}\right] .
\end{aligned}
$$

Since Eq. (6) can be obtained from the conservation of the stress-energy tensor $T_{; v}^{\mu v}=0$, only Eqs. (4) and (5) are actually needed. These can be written in the following forms:

$$
\begin{gathered}
b^{\prime}=8 \pi \rho r^{2}, \\
\Phi^{\prime}=\frac{8 \pi p_{r} r^{3}+b}{2 r(r-b)} .
\end{gathered}
$$

It now becomes apparent that due to the condition $b\left(r_{0}\right)=r_{0}, \Phi^{\prime}(r)$ and hence $\Phi(r)$ are not likely to exist. (There are exceptions, however, for certain special forms of $b(r)$, as shown by Lobo [2].) 
The purpose of this paper is to obtain several complete wormhole solutions by assuming a noncommutative-geometry background in conjunction with an isotropic-pressure condition, discussed in the next section.

\section{NONCOMMUTATIVE GEOMETRY}

An important outcome of string theory is the realization that coordinates may become noncommutative operators on a $D$-brane $[3,4]$. Noncommutativity replaces point-like objects by smeared objects $[5,6,7]$ with the aim of eliminating the divergences that invariably appear in general relativity. As a consequence, spacetime can be encoded in the commutator $\left[\mathbf{x}^{\mu}, \mathbf{x}^{v}\right]=$ $i \theta^{\mu \nu}$, where $\theta^{\mu \nu}$ is an antisymmetric matrix that determines the fundamental cell discretization of spacetime in the same way that Planck's constant discretizes phase space [6]. The smearing can be modeled using a Gaussian distribution of minimal length $\sqrt{\beta}$ instead of the Dirac delta function $[6,8,9,10]$. An equally effective way is to assume that the energy density of the static and spherically symmetric and particle-like gravitational source is given by $[11,12]$

$$
\rho(r)=\frac{\mu \sqrt{\beta}}{\pi^{2}\left(r^{2}+\beta\right)^{2}},
$$

which can be interpreted to mean that the gravitational source causes the mass $\mu$ of a particle to be diffused throughout the region of linear dimension $\sqrt{\beta}$ due to the uncertainty; so $\sqrt{\beta}$ has units of length. Here, it is important to note that the noncommutative effects can be implemented in the Einstein field equations by modifying only the energy-momentum tensor, while leaving the Einstein tensor unchanged. The reason is that, according to [6], a metric field is a geometric structure defined over an underlying manifold. Its strength is measured by curvature, which is a response to the presence of a mass-energy distribution. Here, the key observation is that noncommutativity is an intrinsic property of spacetime (rather than a superimposed geometric structure), thereby affecting the mass-energy and momentum distributions. But the energy-momentum density, in turn, determines the spacetime curvature, which explains why the Einstein tensor can be left unchanged. An important consequence is that the length scales can be macroscopic. We can therefore use Eq. (9) to determine the mass distribution

$$
\int_{0}^{r} 4 \pi\left(r^{\prime}\right)^{2} \rho\left(r^{\prime}\right) d r^{\prime}=\frac{2 M}{\pi}\left(\tan ^{-1} \frac{r}{\sqrt{\beta}}-\frac{r \sqrt{\beta}}{r^{2}+\beta}\right),
$$

where $M$ is now the total mass of the source.

Suppose we return the conservation law $T_{\gamma ; \alpha}^{\alpha}=0$. If $\gamma=r$, then we obtain

$\frac{\partial}{\partial r} T_{r}^{r}=-\frac{1}{2} g^{t t} \frac{\partial g_{t t}}{\partial r}\left(T_{r}^{r}{ }_{r}-T^{t}{ }_{t}\right)-g^{\theta \theta} \frac{\partial g_{\theta \theta}}{\partial r}\left(T_{r}^{r}-T_{\theta}^{\theta}\right)$.
According to [6], to preserve the property $g_{t t}=-g_{r r}^{-1}$, we require that $T^{r}{ }_{r}=T^{t}{ }_{t}=-\rho(r)$, while

$$
T_{\theta}^{\theta}=T_{\phi}^{\phi}=-\rho(r)-\frac{r}{2} \frac{\partial \rho(r)}{\partial r} .
$$

Furthermore, a massive structureless point is replaced by a self-gravitating droplet of anisotropic fluid of density $\rho$, which yields the radial pressure

$$
p_{r}(r)=-\rho(r),
$$

thereby preventing the collapse to a matter point. The tangential pressure is given by

$$
p_{t}(r)=-\rho(r)-\frac{r}{2} \frac{\partial \rho(r)}{\partial r} .
$$

Since the length scales can be macroscopic, we can retain Eq. (13) and then use Eq. (14) to determine

$$
p_{t}(r)=-\rho(r)-\frac{r}{2} \frac{\partial \rho(r)}{\partial r}=p_{r}(r)+\frac{2 \mu r^{2} \sqrt{\beta}}{\pi^{2}\left(r^{2}+\beta\right)^{3}}
$$

by Eq. (9). So, for larger $r$, we have $p_{t}(r) \approx p_{r}(r)$. Since the pressure becomes isotropic, we can write the equation of state in the form

$$
p(r)=-\rho(r) .
$$

In the present context, this is an important observation since Morris-Thorne wormholes are normally characterized by an anisotropic pressure.

\section{A WORMHOLE SOLUTION}

The conservation law $T^{\mu v}{ }_{j v}=0$ yields the following equation:

$$
p_{r}^{\prime}=-\Phi^{\prime} \rho-\Phi^{\prime} p_{r}-\frac{2}{r} p_{r}+\frac{2}{r} p_{t}
$$

From Eq. (16), we obtain

$$
p^{\prime}=-(p+\rho) \Phi^{\prime}
$$

Given the isotropic pressure, we can safely assume the perfect-fluid equation of state

$$
p=\omega \rho,
$$

which, in turn, allows us to consider a cosmological setting. Moreover, in Section 4, we are going to use the more general equation of state

$$
\rho=\frac{1 /|\omega|}{p^{\alpha}}
$$

if $\alpha=-1$, we obtain $p=|\omega| \rho$, which is a special case of Eq. (19). Eq. (20) not only generalizes the equation of state but can also be adapted to a cosmological model 
usually referred to as generalized Chaplygin gas, discussed in Section 5.

Returning now to Eq. (19), if we substitute $p=\omega \rho$ in Eq. (18), we find that $\omega \rho^{\prime}=-\rho(1+\omega) \Phi^{\prime}$ and hence

$$
\Phi^{\prime}=-\frac{\omega}{1+\omega} \frac{\rho^{\prime}}{\rho} .
$$

By integration, we obtain the redshift function

$$
\Phi=-\frac{\omega}{1+\omega}(\ln \rho+\ln c)=-\frac{\omega}{1+\omega} \ln c \rho,
$$

where $c$ is an arbitrary positive constant. Substituting $\rho(r)$ from Eq. (9) yields

$$
\Phi=-\frac{\omega}{1+\omega} \ln \frac{c \mu \sqrt{\beta}}{\pi^{2}\left(r^{2}+\beta\right)^{2}}
$$

so

$$
e^{2 \Phi(r)}=\left[\frac{c \mu \sqrt{\beta}}{\pi^{2}\left(r^{2}+\beta\right)^{2}}\right]^{-\frac{2 \omega}{1+\omega}} .
$$

The next step is to determine the shape function from Eqs. (7) and (9):

$$
\begin{aligned}
b(r)= & \int_{r_{0}}^{r} 8 \pi\left(r^{\prime}\right)^{2} \rho\left(r^{\prime}\right) d r^{\prime} \\
= & \frac{4 m \sqrt{\beta}}{\pi}\left(\frac{1}{\sqrt{\beta}} \tan ^{-1} \frac{r}{\sqrt{\beta}}-\frac{r}{r^{2}+\beta}\right. \\
& \left.-\frac{1}{\sqrt{\beta}} \tan ^{-1} \frac{r_{0}}{\sqrt{\beta}}+\frac{r_{0}}{r_{0}^{2}+\beta}\right)+r_{0}
\end{aligned}
$$

observe that $b\left(r_{0}\right)=r_{0}$, as required. Here, $m$ is the total mass of the source.

One of the requirements of a valid wormhole solution is asymptotic flatness. For the shape function, we evidently have $\lim _{r \rightarrow \infty} b(r) / r=0$. In Eq. (22), we are faced with a more complicated situation: if $-1<\omega<$ 0 , then $\lim _{r \rightarrow \infty} e^{2 \Phi}=0$; if $\omega<-1$ or $\omega \geq 0$, then $\lim _{r \rightarrow \infty} e^{2 \Phi}=+\infty$. Either way, our wormhole spacetime is not asymptotically flat. Accordingly, the wormhole material must be cut off at some $r=a$ and joined to the exterior Schwarzschild solution

$$
\begin{aligned}
d s^{2}= & -\left(1-\frac{2 M}{r}\right) d t^{2}+\frac{d r^{2}}{1-2 M / r} \\
& +r^{2}\left(d \theta^{2}+\sin ^{2} \theta d \phi^{2}\right) .
\end{aligned}
$$

From the shape function $b=b(r)$, we have

$$
\frac{1}{1-\frac{b(a)}{a}}=\frac{1}{1-\frac{2 M}{a}}
$$

So, the effective mass of the wormhole is $M=\frac{1}{2} b(a)$. For the redshift function, we then obtain

$$
e^{2 \Phi(a)}=1-\frac{2 M}{a}=1-\frac{b(a)}{a} .
$$

This junction condition now yields the constant $c$ by letting $r=a$ in Eq. (22):

$$
e^{2 \Phi(a)}=\left[\frac{c \mu \sqrt{\beta}}{\pi^{2}\left(a^{2}+\beta\right)^{2}}\right]^{-\frac{2 \omega}{1+\omega}}=1-\frac{b(a)}{a} .
$$

Solving for $c$, we have

$$
c=\frac{\pi^{2}\left(a^{2}+\beta\right)^{2}}{\mu \sqrt{\beta}}\left(1-\frac{b(a)}{a}\right)^{-\frac{1+\omega}{2 \omega}} .
$$

Using substitution in Eq. (22) and simplification yields the final form

$$
e^{2 \Phi(r)}=\left(\frac{a^{2}+\beta}{r^{2}+\beta}\right)^{-\frac{4 \omega}{1+\omega}}\left(1-\frac{b(a)}{a}\right) .
$$

Observe that at $r=a$, we have indeed

$$
e^{2 \Phi(a)}=1-\frac{b(a)}{a}=1-\frac{2 M}{a} .
$$

The complete solution can now be written as

$$
d s^{2}=-e^{2 \Phi(r)} d t^{2}+\frac{d r^{2}}{1-b(r) / r}+r^{2}\left(d \theta^{2}+\sin ^{2} \theta d \phi^{2}\right),
$$

for $r \leq a$; here $e^{2 \Phi(r)}$ is given by Eq. (29) and $b(r)$ by Eq. (23). For $r>a$,

$$
\begin{aligned}
d s^{2}= & -\left(1-\frac{b(a)}{r}\right) d t^{2}+\frac{d r^{2}}{1-b(a) / r} \\
& +r^{2}\left(d \theta^{2}+\sin ^{2} \theta d \phi^{2}\right) .
\end{aligned}
$$

Since the interior solution, Eq. (31), extends only from $r=r_{0}$ to $r=a$, the solution is valid for all $\omega$, except for $\omega=-1$, as can be seen from Eq. (29).

Returning to the junction surface $r=a$, while our metric is continuous at $r=a$, the derivatives may not be. This behavior needs to be taken into account when discussing the surface stresses. The following forms, proposed by Lobo $[2,13]$, are suitable for this purpose:

$$
\begin{gathered}
\sigma=-\frac{1}{4 \pi a}\left(\sqrt{1-\frac{2 M}{a}}-\sqrt{1-\frac{b(a)}{a}}\right), \\
\mathcal{P}=\frac{1}{8 \pi a}\left(\frac{1-\frac{M}{a}}{\sqrt{1-\frac{2 M}{a}}}-\left[1+a \Phi^{\prime}(a)\right] \sqrt{1-\frac{b(a)}{a}}\right) .
\end{gathered}
$$

Since $b(a)=2 M$, the surface stress-energy $\sigma$ is zero. From Eq. (29), we obtain

$$
\Phi^{\prime}(a)=\frac{\omega}{1+\omega} \frac{4 a}{a^{2}+\beta} .
$$

Using substitution in Eq. (34) and simplification, we get

$$
\mathcal{P}=\frac{1}{8 \pi a \sqrt{1-\frac{2 M}{a}}}\left[\frac{M}{a}+\frac{\omega}{1+\omega} \frac{4 a^{2}}{a^{2}+\beta}\left(-1+\frac{b(a)}{a}\right)\right] .
$$

Due to the condition $b(r)<r$ near the throat, $\mathcal{P}$ is positive whenever

$$
-1<\omega \leq 0
$$




\section{THE GENERALIZED EQUATION OF STATE}

In this section, we return to Eq. (20), restated here for convenience:

$$
\rho=\frac{1 /|\omega|}{p^{\alpha}}, \quad \alpha>-1
$$

From Eq. (18),

$$
\Phi^{\prime}=-\frac{p^{\prime}}{p+\rho}=-\frac{p^{\alpha} p^{\prime}}{p^{\alpha+1}+\frac{1}{|\omega|}} .
$$

By integration, we obtain

$$
2 \Phi=-\frac{2}{\alpha+1} \ln \left[c\left(p^{\alpha+1}+\frac{1}{|\omega|}\right)\right],
$$

and hence

$$
e^{2 \Phi}=\left[c\left(p^{\alpha+1}+\frac{1}{|\omega|}\right)\right]^{-\frac{2}{\alpha+1}}
$$

where

$$
p^{\alpha+1}=\left(\frac{\pi^{2}\left(r^{2}+\beta\right)^{2}}{|\omega| \mu \sqrt{\beta}}\right)^{\frac{\alpha+1}{\alpha}}
$$

from Eq. (9). As before, the resulting wormhole spacetime is not asymptotically flat and needs to be joined to an external Schwarzschild spacetime, i.e.,

$$
\left[c\left(p(a)^{\alpha+1}+\frac{1}{|\omega|}\right)\right]^{-\frac{2}{\alpha+1}}=1-\frac{b(a)}{a} .
$$

Solving for $c$, we get

$$
c=\frac{\left(1-\frac{b(a)}{a}\right)^{-\frac{\alpha+1}{2}}}{p(a)^{\alpha+1}+\frac{1}{|\omega|}}
$$

to be substituted into Eq. (41). The result is

$$
e^{2 \Phi(r)}=\left[\frac{p(r)^{\alpha+1}+\frac{1}{|\omega|}}{p(a)^{\alpha+1}+\frac{1}{|\omega|}}\right]^{-\frac{2}{\alpha+1}}\left(1-\frac{b(a)}{a}\right) .
$$

Using Eq. (42) and reduction, we obtain the final form

$$
e^{2 \Phi(r)}=\left[\frac{\left(\frac{\pi^{2}\left(r^{2}+\beta\right)^{2}}{\mu \sqrt{\beta}}\right)^{\frac{\alpha+1}{\alpha}}|\omega|^{-\frac{1}{\alpha}}+1}{\left(\frac{\pi^{2}\left(a^{2}+\beta\right)^{2}}{\mu \sqrt{\beta}}\right)^{\frac{\alpha+1}{\alpha}}|\omega|^{-\frac{1}{\alpha}}+1}\right]^{-\frac{2}{\alpha+1}}\left(1-\frac{b(a)}{a}\right) .
$$

So, $e^{2 \Phi(a)}=1-\frac{b(a)}{a}$. The shape function is still given by Eq. (23).

We saw earlier that if $\alpha=-1$ in Eq. (38), we recover $p=|\omega| \rho$, a special form of the perfect-fluid equation, but $e^{2 \Phi(r)}$ in Eq. (46) is not defined for $\alpha=-1$. However, the first factor on the right takes on the indeterminate form $1^{\infty}$ at $\alpha=-1$. So, $\lim _{r \rightarrow-1+} e^{2 \Phi(r)}$ can be evaluated by means of L'Hospital's rule. The result is

$$
e^{2 \Phi(r)}=\left(\frac{a^{2}+\beta}{r^{2}+\beta}\right)^{-\frac{4|| \omega \mid}{1+|\omega|}}\left(1-\frac{b(a)}{a}\right),
$$

which is consistent with our previous form, Eq. (29).

\section{THE GENERALIZED CHAPLYGIN- GAS MODEL}

Another equation of state of interest to us is

$$
p=\frac{-K}{\rho^{\alpha}}, \quad 0<\alpha \leq 1
$$

The model with this equation of state is referred to as generalized Chaplygin gas [14, 15, 16, 17]. Cosmologists became interested in this form of the matter when it turned out to be a candidate for combining dark matter and dark energy. To support a wormhole, we must have

$$
K<\frac{1}{\left(8 \pi r_{0}^{2}\right)^{\alpha+1}} .
$$

Suppose we rewrite Eq. (38) in the form

$$
p=\frac{(1 /|\omega|)^{1 / \alpha}}{\rho^{1 / \alpha}} .
$$

Now, assume that $\alpha \geq 1$ and let $\alpha_{1}=1 / \alpha$. Then,

$$
p=\frac{(1 /|\omega|)^{1 / \alpha}}{\rho^{\alpha_{1}}}, \quad 0<\alpha_{1} \leq 1
$$

If we now choose

$$
K=-\left(\frac{1}{|\omega|}\right)^{1 / \alpha}=-|\omega|^{-\frac{1}{\alpha}},
$$

we have a valid equation of state for the Chaplygin model since $K$ can be determined from the free parameter $\omega$.

It now follows directly from Eq. (46) that for the generalized Chaplygin model,

$$
e^{2 \Phi(r)}=\left[\frac{\left(\frac{\pi^{2}\left(r^{2}+\beta\right)^{2}}{\mu \sqrt{\beta}}\right)^{\alpha_{1}+1} K-1}{\left(\frac{\pi^{2}\left(a^{2}+\beta\right)^{2}}{\mu \sqrt{\beta}}\right)^{\alpha_{1}+1} K-1}\right]^{-\frac{2 \alpha_{1}}{\alpha_{1}+1}}\left(1-\frac{b(a)}{a}\right) .
$$

As before, $e^{2 \Phi(a)}=1-\frac{b(a)}{a}$. The shape function is again given by Eq. (23), thereby producing a complete wormhole solution. 


\section{MORE ON NONCOMMUTATIVE GEOMETRY: THE RADIAL TENSION}

While noncommutative geometry was instrumental in obtaining a wormhole solution, its role may extend well beyond the present study. For example, there is an aspect of wormhole physics that goes back to [1] but has not been discussed so far: recalling that the radial tension $\tau(r)$ is the negative of $p_{r}(r)$ and reintroducing $c$ and $G$, the radial tension is given by [1]

$$
\tau(r)=\frac{b(r) / r-2[r-b(r)] \Phi^{\prime}(r)}{8 \pi G c^{-4} r^{2}} .
$$

From this condition, it follows that the radial tension at the throat is

$$
\tau=\frac{1}{8 \pi G c^{-4} r_{0}^{2}} \approx 5 \times 10^{41} \frac{\mathrm{dyn}}{\mathrm{cm}^{2}}\left(\frac{10 \mathrm{~m}}{r_{0}}\right)^{2} .
$$

In particular, for $r_{0}=3 \mathrm{~km}, \tau$ has the same magnitude as the pressure at the center of a massive neutron star. Attributing this outcome to exotic matter is problematical at best since the exotic matter was introduced for a completely different reason: matter is called exotic if it violates the NEC.

It was shown in a recent paper (Kuhfittig [20]) that noncommutative geometry can account for the large radial tension. As an offshoot of string theory, it can therefore be viewed as a foray into quantum gravity, thereby going beyond classical general relativity.

\section{CONCLUSIONS}

The strategy for the theoretical construction of wormholes adopted by Morris and Thorne [1] was to specify the desired geometric properties of the wormhole and then manufacture or search the Universe for matter or fields that would produce the corresponding energymomentum tensor. The purpose of this paper is to determine complete wormhole solutions by starting with a noncommutative-geometry background. Given that the noncommutative effects can be implemented in the Einstein field equations without changing the Einstein tensor, it follows that the length scales can be macroscopic. It was also concluded that for larger $r$, the radial and transverse pressures become equal, thereby yielding the equation of state $p=\omega \rho$, allowing us to consider a cosmological setting. This case is discussed in Section 3. The solution obtained is not asymptotically flat and needs to be cut off and joined to an exterior Schwarzschild solution at the junction surface $r=a$. It was found that the solution is valid for all $\omega \neq-1$. (An examination of the surface stresses has shown, however, that a positive surface pressure at the junction surface is obtained only if $-1<\omega \leq 0$.)

The last part of the paper uses the more general equation of state $\rho=(1 /|\omega|) p^{-\alpha}, \alpha>-1$. This equation of state not only generalizes the previous solution but can also be adapted to another cosmological model, generalized Chaplygin gas. The result is two additional complete wormhole solutions.

Previous studies $[2,18]$ have shown that wormhole solutions exist for $\omega<-1$ since, in a cosmological setting, we are dealing with phantom energy, which is known to violate the null energy condition. While fulfilling the primary prerequisite for the existence of wormholes, this approach yields neither the shape nor the redshift functions. Similarly, a noncommutativegeometry background alone is sufficient for determining $b=b(r)$, as shown in [19], but it does not yield the redshift function $\Phi=\Phi(r)$.

As a final remark, Section 6 reiterates another aspect of noncommutative geometry, the ability to account for the enormous radial tension in a typical traversable wormhole, discussed in [20].

\section{CONFLICTS OF INTEREST}

The author declares that there are no conflicts of interest regarding the publication of this paper.

\section{References}

[1] M. S. Morris and K. S. Thorne, Am. J. Phys. 56, 395412 (1988).

[2] F. S. N. Lobo, Phys. Rev. D 71, 0844011 (2005).

[3] E. Witten, Nucl. Phys. B 460, 335-350 (1996).

[4] N. Seiberg and E. Witten, J. High Energy Phys. 9909, 032 (1999).

[5] A. Smailagic and E. Spallucci, J. Phys. A 36, L-467L-471 (2003).

[6] P. Nicolini, A. Smailagic and E. Spallucci, Phys. Lett. B 632, 547-551 (2006).

[7] P. Nicolini and E. Spallucci, Class. Quant. Grav. 27, 015019 (2010).

[8] M. Rinaldi, Class. Quant. Grav. 28, 105022 (2011).

[9] F. Rahaman, P. K. F. Kuhfittig, S. Ray and S. Islam, Phys. Rev. D 86, 106101 (2012).

[10] P. K. F. Kuhfittig, Int. J. Pure Appl. Math. 89, 401-408 (2013).

[11] K. Nozari and S. H. Mehdipour, Class. Quant. Grav. 25, 175015 (2008).

[12] J. Liang and B. Liu, EPL 100, 30001 (2012).

[13] F. S. N. Lobo, Class. Quant. Grav. 21, 4811 (2004).

[14] F. S. N. Lobo, Phys. Rev. D 73, 064028 (2006).

[15] A. Y. Kamenshchik, U. Moschella and V. Pasquier Phys. Lett. B 511, 265-268 (2001).

[16] M. C. Bento, O. Bertolami and A. A. Sen, Phys. Rev. D 66, 043507 (2002).

[17] P. K. F. Kuhfittig, arXiv: 0802.365.

[18] S. V. Sushkov, Phys. Rev. D 71, 043520 (2005).

[19] P. K. F. Kuhfittig, Int. J. Mod. Phys. D 24, 1550023 (2015).

[20] P. K. F. Kuhfittig, Eur. Phys. J. Plus 135, 510 (2020). 